\title{
Guest Editorial: Multimedia Data Sensing and Analyzing of Surveillance Systems
}

\author{
Xiangfeng Luo ${ }^{1} \cdot$ Yunhuai Liu ${ }^{2} \cdot$ Zheng $\mathrm{Xu}^{2} \cdot{\mathrm{Qing} \mathrm{Li}^{3}}^{3}$
}

Published online: 3 August 2015

(C) Springer Science+Business Media New York 2016

Big data is an emerging paradigm applied to datasets whose size is beyond the ability of commonly used software tools to capture, manage, and process the data within a tolerable elapsed time. With the pervasive of the definition of the smart city, the surveillance system, huge number of video surveillance devices such as surveillance cameras. Understanding the semantics of surveillance device has been an important component in many video-based applications. Manual annotation and tagging has been considered as a reliable source of video semantics. Unfortunately, manual annotation is time-consuming and expensive when dealing with huge scale of video data. However, the semantic gap between semantics and video visual appearance is still a challenge towards automated ontology-driven video annotation. Thus, automatically understanding raw videos solely based on their visual appearance becomes an important yet challenging problem. Thus, it is important to accurately describe the video content and enable the organizing and searching potential videos.

The submitted manuscripts were reviewed by experts from both academia and industry. After two rounds of reviewing, the highest quality manuscripts were accepted for this special issue. Totally, we have received 27 manuscripts and 14 papers are accepted. The accepted rate is about $50 \%$. This special issue will be published by Multimedia Tools and Applications as special issues.

The paper by H. Wang et al. [8] proposed a novel color-based road detection method based on a boundary ratio prior, with which we are able to infer the confidence of a certain image region belonging to the road class. The paper by J. Yu et al. [11] illustrates the 3D facial animation by combining the parameterized model and muscular model. 3D hair was

Xiangfeng Luo luoxf303@163.com

1 Shanghai University, Shanghai, China

2 The Third Research Institute of the Ministry of Public Security, Shanghai, China

3 City University of Hong Kong, Kowloon Tong, Hong Kong 
synthesized based on the hair detection result in video. 3D coding/decoding result of foreground and 2D coding/decoding result of background were stitched seamlessly. The paper by J. Lei et al. [4] proposed a robust k-means algorithm that can automatically split and merge clusters which incorporates the new ideas in dealing with huge scale of video data. The paper by M. Zhang et al. [7] aim at revealing the regional difference of coast land use in the Bohai Sea, series remote sensing images of HJ-1ACCD obtained in July of 2013 were employed to monitor land use in $5 \mathrm{~km}$ coastal zone of 13 regions around the Bohai Sea. The paper by Y. Zhang et al. [13] introduce the down-sampling and up-sampling to form a pair of re-sampling filter, called RSFP, serving as an approximation of the current layer of pyramid data, can be used to evaluate the effect of the down-sampling filter. The paper by Y. Zhang et al. [14] proposed a method based on reorganization of blocked discrete cosine transform (RBDCT) to separate the mixed images. The paper by C. Wang et al. [1] presents a completely rely on spatial geometry calculations to achieve the user's line of sight placement calculation method. The paper by S. Wu et al. [9] introduces an improved classification method based on sparse representation by representing the test samples through a dictionary. The paper by $\mathrm{Z}$. Xu et al. [10] introduced a semantic-based model named video structural description (VSD) for representing and organizing the content in videos. The paper by B. Zhai et al. [12] presents an adaptive motion filtering algorithm with feedback correction. The paper by C. Fan et al. [2] proposed take association relation and the main steps of building such a concept semantic space on text database are discussed in detail. According to the features of target region in images to be processed, D. Fan et al. [3] presents a solution framework based on artificial neural network (ANN). An integrated de-noising method, based on assemble of multiple image smoothing filters, is proposed in [6]. A novel multi-instance multi-label learning algorithm is proposed by C. Liu et al. [5] modeling instance correlations in each bag.

Acknowledgments The guest editors would like to thank Prof. Borivoje Furht who is the editor in chief of Multimedia Tools and Applications. His help and trust is the most important thing for the success of this SI. The guest editors would like to thank the reviewers for their high-quality reviews, which provided insightful and constructive feedback to the authors of the papers.

\section{References}

1. Changyuan W, Li W, Pengxiang X. The line of sight to estimate method based on stereo vision. Multimed Tools Appl. doi:10.1007/s11042-016-3283-8.

2. Fan C, Wang L, Liu P, Lu K, Liu D. Compressed sensing based remote sensing image reconstruction via employing similarities of reference images. Multimed Tools Appl. doi:10.1007/s11042-015-3004-8.

3. Fan $\mathrm{D}$ et al. Extraction of target region in lung immunohistochemical image based on artificial neural network. Multimed Tools Appl. doi:10.1007/s11042-016-3459-2.

4. Lei J, Jiang T, Wu K, Du H, Zhu G, Wang Z. Robust K-means algorithm with automatically splitting and merging clusters and its applications for surveillance data. Multimed Tools Appl. doi:10.1007/s11042-016-3322-5.

5. Liu $\mathrm{C}$ et al. A multi-instance multi-label learning algorithm based on instance correlations. Multimed Tools Appl. doi:10.1007/s11042-016-3494-Z.

6. Shi Z, Xu B, Zheng X, Zhao M. An integrated method for ancient Chinese tablet images de-noising based on assemble of multiple image smoothing filters. Multimed Tools Appl. doi:10.1007/s11042-016-3421-3.

7. Suo A, Lin Y, Zhang M. Regional difference of coastal land use around the Bohai sea based on remote sensing images. Multimed Tools Appl. doi:10.1007/s11042-016-3334-1.

8. Wang H, Ren M, Yang J. Capitalizing on the boundary ratio prior for road detection. Multimed Tools Appl. doi:10.1007/s11042-016-3280-y. 
9. Wu S, Chen H, Bai Y, Zhu G. A remote sensing image classification method based on sparse representation. Multimed Tools Appl. doi:10.1007/s11042-016-3320-7.

10. $\mathrm{Xu} \mathrm{Z,} \mathrm{Hu} \mathrm{C,} \mathrm{Mei} \mathrm{L.} \mathrm{Video} \mathrm{structured} \mathrm{description} \mathrm{technology} \mathrm{based} \mathrm{intelligence} \mathrm{analysis} \mathrm{of} \mathrm{surveillance}$ videos for public security applications. Multimed Tools Appl. doi:10.1007/s11042-015-3112-5.

11. Yu J, Luo C, Yu L, Li L, Wang Z. Facial video coding/decoding at ultra-low bit-rate: a 2D/3D model-based approach. Multimed Tools Appl. doi:10.1007/s11042-016-3368-4.

12. Zhai B, Zheng J, Li B. Digital image stabilization based on adaptive motion filtering with feedback correction. Multimed Tools Appl. doi:10.1007/s11042-015-3183-3.

13. Zhang Y, Zhang M, Wang J, Zhang G. ICRS: inter-layer compression method combined with generation of a spatial image pyramid. Multimed Tools Appl. doi:10.1007/s11042-015-3193-1.

14. Zhang Y, Yang D, Qi R, Gong Z. Blind image separation based on reorganization of block DCT. Multimed Tools Appl. doi:10.1007/s11042-016-3397-z. 